\title{
Study of the Stability and Accuracy of the Eccentricity Identification Algorithm in Fast-Revolving Rotor Balancing Problems
}

\author{
A. V. Goroshko ${ }^{a}$, V. P. Royzman ${ }^{a}$, and O. V. Barmina ${ }^{b}$ \\ ${ }^{a}$ Khmelnitskyi National University, Khmelnitskyi, Ukraine \\ ${ }^{b}$ Institute for Machine Science, Russian Academy of Sciences, Malyi Khariton'evskii per. 4, Moscow, 101990 Russia \\ e-mail: royzman_V@mail.ru
}

Received February 19, 2015

\begin{abstract}
Problems related to the stability of the eccentricity identification algorithm in the problem of balancing flexible rotors are considered. The accuracy of the algorithm developed for identification by static influence coefficients is studied. The physical meaning of the condition number in the eccentricity identification problem is revealed. Optimal relationships are established between the balancing accuracy, the precision of measurement instrumentation, and the dynamic model of the system under consideration.
\end{abstract}

DOI: $10.3103 / \mathrm{S} 1052618816030079$

\section{INTRODUCTION}

It was noted in [1] that parametric identification of a flexible rotor involved the solution to the inverse problem of the vibration theory wherein the forces and eccentricities causing a dynamic system to move should be reconstructed from the actually observed and recorded motion of the system. In this problem, the question of identification of the dynamic system arises, i.e., the possibility, in principle, to unambiguously determine the vectors of unknown quantities at hand, and then, the reliability of the identification-possible errors in determining these quantities, which stem from the fact that the objective identification property frequently turns out to have been incompletely revealed due to the inadequacy of the identification algorithms used.

\section{FORMULATION OF THE PROBLEM}

Let us consider the problem of the stability of balancing as regards the correctness of the chosen identification algorithm or the adopted dynamic model of the rotor under consideration, this being to imply the fact that small variations in the input-experimentally found-parameters cause insignificant changes in the values of the eccentricities and disbalances being identified and, as a result, in the values of the correction masses needed. Such a notion of stability can be defined more rigorously as follows. Let the input parameters $a_{1}, a_{2}, \ldots, a_{n}$ be related to the sought $x_{1}, x_{2}, \ldots, x_{k}$ by a scalar or vector equation of the form $f\left(a_{1}\right.$, $\left.a_{2}, \ldots, a_{n}, x_{1}, x_{2}, \ldots, x_{k}\right)=0, n, k \in \mathbf{N}$.

The chosen model is said to be stable if for an arbitrary number $\varepsilon>0$ there exists $\delta>0$ such that as soon as $\|\mathbf{a}\|<\delta$, then $\|\mathbf{x}\|<\varepsilon$, where $\mathbf{x}$ is the vector with the coordinates $x_{1}, x_{2}, \ldots, x_{k}$, a is a vector with the coordinates $a_{1}, a_{2}, \ldots, a_{n}$, and the symbol \|\| denotes an arbitrary vector norm. We also defined in a similar way stability with reference to a single input parameter, or a group of parameters, rather than all of them, $\mathbf{a}$ in this case being considered a vector wherein all the coordinates, except for the chosen ones, are constant quantities.

In view of the fact that the majority of eccentricity identification methods involve solving systems of linear algebraic equations, let us consider the stability and accuracy of eccentricity identification in the case where for the static influence coefficients, because here the physical meaning of the phenomenon is clearly manifested. 


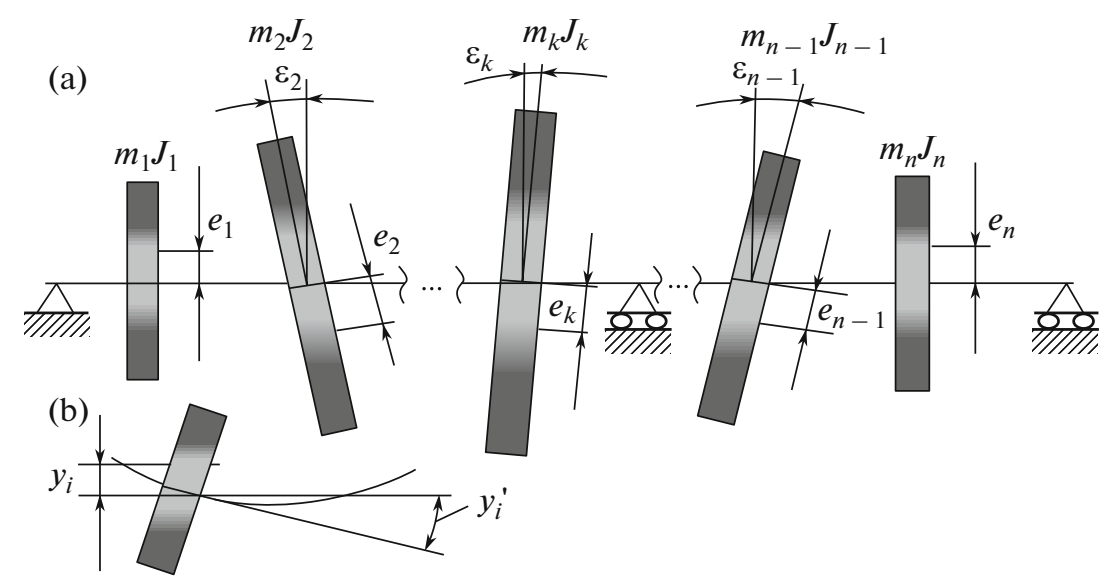

Fig. 1. Schematic diagram of multiple-mass rotor.

\section{STUDY OF ECCENTRICITY IDENTIFICATION BY STATIC INFLUENCE COEFFICIENTS}

It was demonstrated in [1] that the motion of a flexible rotor relative to a coordinate system rotating together with it and having one of its axes coincident with the rotor axis is described by a Fredholm equation of the second kind. This equation can be approximated to within any given accuracy by a system of linear algebraic equations [2], which means that a rotor with any distribution of parameters can be approximated by a rotor consisting of a weightless shaft carrying $n$ disks with masses $m_{i}$ and equatorial moments of inertia $J_{i}, i=1,2, \ldots, n$. By virtue of their manufacturing errors, each of the disks features radial and angular eccentricities, $e_{i}$ and $\varepsilon_{i}$, respectively, i.e., deviations of the disk axis from the axis passing through the centers of inertia (Fig. 1a). During rotation, the shaft acquires deflections $y_{i}$ and rotation angles $y_{i}^{\prime}$ (Fig. 1b).

With the gyroscopic moments disregarded, the Fredholm equation of the second kind is replaced by a matrix equation of the form

$$
\mathbf{y}=\mathbf{A}(\mathbf{y}+\mathbf{e}) \omega^{2}
$$

where $\mathbf{y}$ and $\mathbf{e}$ are vectors of length $n$ and $\mathbf{A}$ is an $n \times n$ square matrix.

In this equation, the coordinates of vector $\mathbf{y}$ are the rotor shaft deflections at the seats of the disks, and those of vector $\mathbf{e}$, the eccentricities of these disks. The elements of matrix $\mathbf{A}$ are the products of the static coefficients of influence on the masses of the relevant disks [3]. The value of the static influence coefficient, $\alpha_{i j}$, is equal to the deflection of the rotor shaft in the $i$ th section due to the action of a unit force in the $j$ th section, $i, j=1,2, \ldots, n$.

The model described is identifiable; i.e., according to Cramer's rule, its unique solution exists. However, the same model is of practical use only if small errors arising upon variations of the experimentally found quantities (the deflections y) entering into Eq. (1) will cause, during the course of its solution, only small errors in the determination of the eccentricities, i.e., if the model is resistant to these factors.

Let the system

$$
\mathbf{y}+\Delta \mathbf{y}=\left(\omega^{2}+\Delta \omega^{2}\right)(\mathbf{A}+\Delta \mathbf{A})(\mathbf{y}+\mathbf{e}+\Delta \mathbf{y}+\Delta \mathbf{e})
$$

be actually solved instead of system (1); i.e., all the terms of the original equation have some unknown errors depending on the precision of the control instrumentation used, these errors being small compared to the quantities measured.

Let us single out the principal error term by subtracting Eq. (1) from Eq. (2), in doing so disregarding the terms of second and higher orders of smallness:

$$
\Delta \mathbf{y}=\omega^{2} \Delta \mathbf{A}(\mathbf{y}+\mathbf{e}) \Delta \omega^{2} \mathbf{A}(\mathbf{y}+\mathbf{e})+\omega^{2} \mathbf{A} \Delta \mathbf{y}+\omega^{2} \mathbf{A} \Delta \mathbf{e} .
$$

From Eq. (1) we have $\mathbf{y}+\mathbf{e}=\mathbf{A}^{-1} \mathbf{y} / \omega^{2}$. Substituting this expression into the preceding equation, we obtain

$$
\Delta \mathbf{e}=\frac{-\mathbf{A}^{-1} \Delta \mathbf{y} \mathbf{A}^{-1} \mathbf{y}}{\omega^{2}}-\frac{\mathbf{A}^{-1} \mathbf{y} \Delta \omega^{2}}{\omega^{4}}-\Delta \mathbf{y}+\frac{\mathbf{A}^{-1} \Delta \mathbf{y}}{\omega^{2}} .
$$


Hence, in an arbitrary norm, we have

$$
\|\Delta \mathbf{e}\| \leq\left\|\mathbf{A}^{-1}\right\|\|\mathbf{E}-\mathbf{A}\|\|\Delta \mathbf{y}\|+\frac{\left\|\mathbf{A}^{-1}\right\|^{2}\|\Delta \mathbf{A}\|\|\mathbf{y}\|}{\omega^{2}}+\frac{\left\|\mathbf{A}^{-1}\right\|\left\|\Delta \omega^{2}\right\|\|\Delta \mathbf{y}\|}{\omega^{4}} .
$$

From Eq. (1) we can easily obtain $\mathbf{y} / \omega^{2}-\mathbf{A y}=\mathbf{A e},\left(\mathbf{E} / \omega^{2}-\mathbf{A}\right) \mathbf{y}=\mathbf{A e}, \mathbf{y}=\left(\mathbf{E} / \omega^{2}-\mathbf{A}\right)^{-1} \mathbf{A e}$. Therefore, $\|\mathbf{y}\| \leqslant\left\|\left(\frac{\mathbf{E}}{\omega^{2}}-\mathbf{A}\right)^{-1}\right\|\|\mathbf{A}\|\|\mathbf{e}\|$. Hence,

$$
\|\mathbf{e}\| \geq \frac{\|\mathbf{y}\|}{\|\mathbf{A}\|}\left\|\left(\frac{\mathbf{E}}{\omega^{2}}-\mathbf{A}\right)\right\|
$$

Dividing expression (3) by (4), we obtain

$$
\begin{gathered}
\frac{\|\Delta \mathbf{e}\|}{\|\mathbf{e}\|} \leq \frac{\|\Delta \mathbf{y}\|}{\|\mathbf{y}\|}\left\|\mathbf{A}^{-1}\right\|\|\mathbf{A}\|\left\|\frac{\mathbf{E}}{\omega^{2}}-\mathbf{A}\right\|\left\|\left(\frac{\mathbf{E}}{\omega^{2}}-\mathbf{A}\right)^{-1}\right\|+\frac{\left\|\mathbf{A}^{-1}\right\|^{2}\|\mathbf{A}\|^{2}}{\omega^{2}} \\
\times\left\|\left(\frac{\mathbf{E}}{\omega^{2}}-\mathbf{A}\right)^{-1}\right\| \frac{\|\Delta \mathbf{A}\|}{\|\mathbf{A}\|}+\frac{\left\|\mathbf{A}^{-1}\right\|\|\mathbf{A}\|}{\omega^{2}}\left\|\left(\frac{\mathbf{E}}{\omega^{2}}-\mathbf{A}\right)^{-1}\right\| \frac{\Delta \omega^{2}}{\omega^{2}} .
\end{gathered}
$$

It is a well-established fact that for an arbitrary square matrix $\mathbf{A}$, the matrix norm multiplied by its inverse yields the matrix condition number cond(A); i.e., cond (A) $=\|\mathbf{A}\| \times\|\mathbf{A}\|^{-1} \geqslant 1$ [4]. For this reason, $\left\|\mathbf{E} / \omega^{2}-\mathbf{A}\right\| \times\left\|\left(\mathbf{E} / \omega^{2}-\mathbf{A}\right)^{-1}\right\| \geqslant 1$ and

$$
\begin{aligned}
\frac{\|\Delta \mathbf{e}\|}{\|\mathbf{e}\|} \leq \operatorname{cond}(\mathbf{A}) \operatorname{cond}\left(\frac{\mathbf{E}}{\omega^{2}}-\mathbf{A}\right) \frac{\|\Delta \mathbf{y}\|}{\|\mathbf{y}\|}+\frac{\operatorname{cond}(\mathbf{A})^{2}}{\omega^{2}}\left[\operatorname{cond}\left(\frac{\mathbf{E}}{\omega^{2}}-\mathbf{A}\right) /\left\|\frac{\mathbf{E}}{\omega^{2}}-\mathbf{A}\right\|\right] \| \frac{\|\mathbf{A}\|}{\|\mathbf{A}\|} \\
+\frac{\operatorname{cond}(\mathbf{A})}{\omega^{2}}\left[\operatorname{cond}\left(\frac{\mathbf{E}}{\omega^{2}}-\mathbf{A}\right)\left\|\frac{\mathbf{E}}{\omega^{2}}-\mathbf{A}\right\|^{-1}\right] \frac{\Delta \omega^{2} \mid}{\omega^{2}} .
\end{aligned}
$$

We can see from inequality (6) that balancing with the static influence coefficients is theoretically stable.

However, one can hardly believe that rotor shaft deflections and their measurement errors can be smaller than any given quantities, if for no other reason than the lack of means to measure them in this case. Therefore, when investigating stability problems, one should restrict oneself to the precision of the available instrumentation for measuring deflections, speeds, influence coefficients, and so on.

In particular cases, we can easily obtain the following upper-bound estimates of the relative error of eccentricities:

$$
\begin{gathered}
\|\Delta \mathbf{e}\| /\|\mathbf{e}\| \leq \operatorname{cond}(\mathbf{A}) \operatorname{cond}\left(\mathbf{E} / \omega^{2}-\mathbf{A}\right)\|\Delta \mathbf{y}\| /\|\mathbf{y}\| \quad \text { at } \quad \Delta \mathbf{A}=0 \quad \text { and } \quad \Delta \omega^{2}=0, \\
\|\Delta \mathbf{e}\| /\|\mathbf{e}\| \leq \frac{1}{\omega^{2}} \frac{\operatorname{cond}(\mathbf{A}) \operatorname{cond}\left(\mathbf{E} / \omega^{2}-\mathbf{A}\right)}{\left\|\mathbf{E} / \omega^{2}-\mathbf{A}\right\|} \frac{\left|\Delta \omega^{2}\right|}{\omega^{2}} \quad \text { at } \quad \Delta \mathbf{y}=0 \quad \text { and } \quad \Delta \mathbf{A}=0, \\
\|\Delta \mathbf{e}\| /\|\mathbf{e}\| \leq \frac{1}{\omega^{2}}[\operatorname{cond}(\mathbf{A})]^{2} \frac{\operatorname{cond}\left(\mathbf{E} / \omega^{2}-\mathbf{A}\right)}{\left\|\mathbf{E} / \omega^{2}-\mathbf{A}\right\|} \frac{\|\Delta \mathbf{A}\|}{\|\mathbf{A}\|} \quad \text { at } \quad \Delta \mathbf{y}=0 \quad \text { and } \quad \Delta \omega^{2}=0 .
\end{gathered}
$$

Relation (7) expresses the stability of the theoretical model with respect to the rotor shaft deflection; relation (8), that with respect to the shaft rotation frequency; and relation (9), that with respect to the properties of matrix $\mathbf{A}$.

It is known from matrix theory that the condition number of a matrix indicates the maximum number of times the relative error of a result can exceed the relative error of the original information.

Let us clarify the physical meaning of the condition numbers of matrices $\mathbf{A}$ and $\mathbf{E} / \omega^{2}-\mathbf{A}$. It is well known [4] that the condition number of a matrix in the Euclidean norm can be expressed as the ratio between its maximum and minimum eigenvalues; i.e.,

$$
\operatorname{cond}(\mathbf{A})=\left(\max _{i}\left|\lambda_{i}\right|\right) /\left(\min _{i}\left|\lambda_{i}\right|\right), \quad \operatorname{cond}\left(\mathbf{E} / \omega^{2}-\mathbf{A}\right)=\left(\max _{i}\left|\mu_{i}\right|\right) /\left(\min _{i}\left|\mu_{i}\right|\right) .
$$


The eigenvalues of the matrix

$$
\mathbf{A}=\left(\begin{array}{cccc}
m_{1} \alpha_{11} & m_{2} \alpha_{12} & \ldots & m_{n} \alpha_{1 n} \\
m_{1} \alpha_{21} & m_{2} \alpha_{22} & \ldots & m_{n} \alpha_{2 n} \\
\ldots & \ldots & \ldots & \ldots \\
m_{1} \alpha_{n 1} & m_{2} \alpha_{n 2} & \ldots & m_{n} \alpha_{n n}
\end{array}\right)
$$

are found such that the determinant lacking angular velocity is equal to zero:

$$
\operatorname{det}\left|\mathbf{A}-\lambda_{i} \mathbf{E}\right|=0 \text {, }
$$

where $\mathbf{E}$ is unity matrix.

The squared rotation eigenfrequencies of the above-described system are the roots of the equation

$$
\operatorname{det}\left|\omega_{i}^{2} \mathbf{A}-\mathbf{E}\right|=0 \text {. }
$$

Comparing expressions (10) and (11), we can see that $\lambda_{i}=1 / \omega_{i}^{2}, i=1,2, \ldots, n$. Then, obviously, $\lambda_{\max }=$ $1 / \omega_{1}^{2}$ and $\lambda_{\min }=1 / \omega_{n}^{2}$, and so,

$$
\operatorname{cond}(\mathbf{A}) \geq \omega_{n}^{2} / \omega_{1}^{2} .
$$

Consequently, the lower-bound estimate of the condition number of the matrix $\mathbf{A}$ is formed by the ratio between the squares of the maximum and minimum rotation eigenfrequencies of the rotor represented by the given discrete model.

Naturally, the greater the size $n$, the higher this model conforms to reality, but then, as seen from (12), cond(A) and, hence, the error of the solution also increase. Consequently, on the one hand, approximating the actual rotor by a great number of concentrated masses, we bring the theoretical scheme closer to the actual construction and thus improve the accuracy in finding the stiffnesses or influence coefficients, as well as that of the calculation procedure as a whole; however, on the other hand, as $n$ is increased, the condition number increases, and so do the calculation errors. This contradiction results from the imperfection of the identification algorithm. These should be taken into account when justifying the acceptability of the above-described identification technique, with due regard for the accuracy of the given balancing method, while selecting a reasonable number of disks to approximate the actual rotor [5]. This emphasizes the fact that the most acceptable and stable are relatively simple theoretical models using exact, experimentally found values of the parameters, such as stiffnesses, masses, deflections, etc., corresponding to the given theoretical scheme.

Now let us reveal the physical meaning of the condition number of matrix $\mathbf{E} / \omega^{2}-\mathbf{A}$ :

$$
\operatorname{det}\left|\mathbf{E} / \omega^{2}-\mathbf{A}-\mu \mathbf{E}\right|=0 .
$$

We reduce equation (13) to the form

$$
0=\operatorname{det}\left|\mathbf{A}+\left(\mu-1 / \omega^{2}\right) \mathbf{E}\right|=\operatorname{det}\left|\mathbf{A}-\left(1 / \omega^{2}-\mu\right) \mathbf{E}\right| .
$$

We found earlier that the roots of Eq. (14) have the form $1 / \omega^{2}-\mu_{i}=1 / \omega_{i}^{2}, i=1,2, \ldots, n$. Hence $\mu_{i}=$ $1 / \omega^{2}-1 / \omega_{i}^{2}$ and

$$
\operatorname{cond}\left(\mathbf{E} / \omega^{2}-\mathbf{A}\right) \geq\left(\max _{i}\left|\omega^{-2}-\omega_{i}^{-2}\right|\right) /\left(\min _{i}\left|\omega^{-2}-\omega_{i}^{-2}\right|\right) .
$$

It can be seen that the maximum and minimum values of $\mu_{i}$ depend on the discrete model selected; i.e., on the number $n$ of the masses and rotation frequency at which the measurement and balancing procedures are executed.

Assuming that $\omega_{1}^{2} \leqslant \omega_{2}^{2} \leqslant \ldots \leqslant \omega_{n}^{2}$, inequality (15) can be rendered more exact as follows. If $0<\omega^{2}<$ $\omega_{1}^{2}$, then $\left|1 / \omega^{2}-1 / \omega_{i}^{2}\right|=1 / \omega^{2}-1 / \omega_{i}^{2}, i=1,2, \ldots, n$. In that case,

$$
\operatorname{cond}\left(\mathbf{E} / \omega^{2}-\mathbf{A}\right) \geq \frac{\omega^{-2}-\omega_{n}^{-2}}{\omega^{-2}-\omega_{1}^{-2}}=\frac{\omega_{n}^{2}-\omega^{2}}{\omega_{1}^{2}-\omega^{2}} \frac{\omega_{1}^{2}}{\omega_{n}^{2}} .
$$




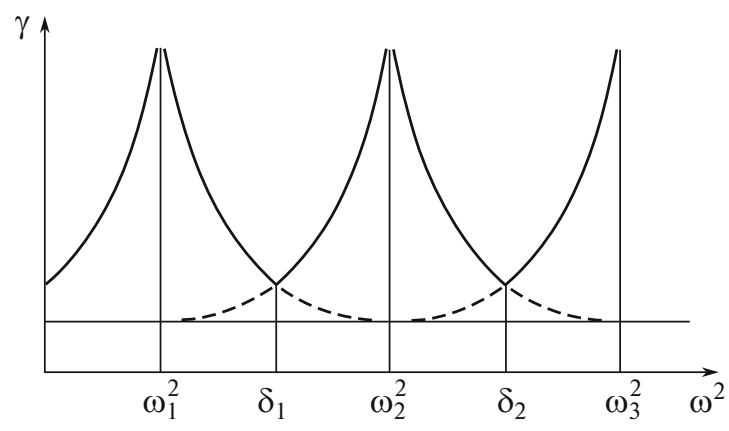

Fig. 2. Product of condition numbers of matrices $\mathbf{A}$ and $\mathbf{E} / \omega^{2}-\mathbf{A}$ as function of rotor rotation frequency in balancing.

If $\omega_{k}^{2} \leqslant \omega^{2} \leqslant \omega_{k+1}^{2}$, the magnitudes of eigenvalues $\mu_{i}$ have the form $\left|\mu_{1}\right|=1 / \omega_{1}^{2}-1 / \omega^{2}, \ldots,\left|\mu_{k}\right|=$ $1 / \omega_{k}^{2}-1 / \omega^{2},\left|\mu_{k+1}\right|=1 / \omega^{2}-1 / \omega_{k+1}^{2},\left|\mu_{n}\right|=1 / \omega^{2}-1 / \omega_{n}^{2}$.

Obviously $\max \left|\mu_{i}\right|$ can occur either at $i=1$ or $i=n$, and $\min \left|\mu_{i}\right|$ in the given range of angular velocities can occur either at $i=k$, or at $i=k+1,\left|\mu_{k}\right|$ being smaller than $\left|\mu_{k+1}\right|$ at $1 / \omega_{k}^{2}-1 / \omega^{2} \leqslant 1 / \omega^{2}-1 / \omega_{k+1}^{2}$, i.e., at $\omega^{2}<2 \omega_{k}^{2} \omega_{k+1}^{2} /\left(\omega_{k}^{2}+\omega_{k+1}^{2}\right)=\delta_{k}$, otherwise $\left|\mu_{k}\right| \geqslant\left|\mu_{k+1}\right|$.

Thus,

$$
\begin{gathered}
\operatorname{cond}\left(\frac{\mathbf{E}}{\omega^{2}}-\mathbf{A}\right) \geq \frac{\left|\omega^{-2}-\omega_{i}^{-2}\right|}{\left|\omega^{-2}-\omega_{k}^{-2}\right|} \quad \text { for } \quad \omega_{k}^{2}<\omega^{2}<\delta_{k}, \quad i=1 \quad \text { or } \quad i=n, \\
\operatorname{cond}\left(\frac{\mathbf{E}}{\omega^{2}}-\mathbf{A}\right) \geq \frac{\left|\omega^{-2}-\omega_{i}^{-2}\right|}{\left|\omega^{-2}-\omega_{k+1}^{-2}\right|} \quad \text { for } \quad \delta_{k}<\omega^{2}<\omega_{k+1}^{2}, \quad i=1 \quad \text { or } \quad i=n .
\end{gathered}
$$

That is,

$$
\begin{gathered}
\operatorname{cond}(\mathbf{A}) \operatorname{cond}\left(\frac{\mathbf{E}}{\omega^{2}}-\mathbf{A}\right) \geq \frac{\left|\omega_{i}^{2}-\omega^{2}\right|}{\left|\omega_{k}^{2}-\omega^{2}\right|} \frac{\omega_{k}^{2}}{\omega_{i}^{2}} \frac{\omega_{n}^{2}}{\omega_{1}^{2}}=\gamma \\
\text { for } \omega_{k}^{2}<\omega^{2}<\delta_{k}, \quad i=1 \quad \text { or } \quad i=n, \\
\operatorname{cond}(\mathbf{A}) \operatorname{cond}\left(\frac{\mathbf{E}}{\omega^{2}}-\mathbf{A}\right) \geq \frac{\left|\omega_{i}^{2}-\omega^{2}\right|}{\left|\omega_{k+1}^{2}-\omega^{2}\right|} \frac{\omega_{k+1}^{2}}{\omega_{i}^{2}} \frac{\omega_{n}^{2}}{\omega_{1}^{2}}=\gamma \\
\text { for } \delta_{k}<\omega^{2}<\omega_{k+1}^{2}, \quad i=1 \quad \text { or } \quad i=n .
\end{gathered}
$$

The plot of $\gamma=\operatorname{cond}(\mathbf{A}) \times \operatorname{cond}\left(\mathbf{E} / \omega^{2}-\mathbf{A}\right)$ as a function of $\omega^{2}$ (Fig. 2) shows that the least stable rotation frequency regions in balancing are those in the neighborhood of the critical frequencies. The selected model will be most stable at angular velocities determined by the expression $\omega=\omega_{k} \times$ $\omega_{k+1} \sqrt{2 /\left(\omega_{k}^{2}+\omega_{k+1}^{2}\right)}$.

Moreover, the identification algorithm (and the balancing technique at the same time) is the more stable, the more flexible the rotor. Indeed, let us consider the growth rate of $\gamma$, e.g., in the subcritical region of the rotor rotation frequencies:

$$
\frac{d \gamma}{d \omega^{2}}=\frac{\omega_{n}^{2}-\omega_{1}^{2}}{\left(\omega_{1}^{2}-\omega^{2}\right)^{2}}=\frac{\omega_{n}^{2} / \omega_{1}^{2}-1}{\left(\omega_{1}^{2}-\omega^{2}\right)^{2}} \cdot \omega_{1}^{2}>0
$$

i.e., the lower the ratio $\omega_{n}^{2} / \omega_{1}^{2}$, or the higher the flexibility of the rotor, the lower the growth rate of the calculation error, and, vice versa, the stiffer the rotor, the higher the rate of growth of the error with increasing rotation frequency in balancing. 


\section{PRACTICAL IMPORTANCE OF THE RESULTS OF THEORETICAL IMVESTIGATIONS}

Formulas (7)-(9) make it possible to solve some purely practical problems. Specifically, given the accuracy class of rotor balancing, which determines quantity $\Delta \mathbf{e} / \mathbf{e}$ and the selected calculation model, hence, also cond(A)cond $\left(\mathbf{E} / \omega^{2}-\mathbf{A}\right)$, formula (7) makes it possible to find $\Delta \mathbf{y} / \mathbf{y}$, i.e., the relative error of instruments for measuring rotor deflections, and select suitable instruments.

If one has at one's disposal appropriate measuring instruments and a properly selected model of the rotor, then one will be able to determine the attainable accuracy of balancing.

If the accuracy class of balancing is specified and the appropriate instruments for measuring deflections are available, it will then be possible to find a calculation model capable of ensuring the necessary balancing accuracy.

The same reasoning pertains to formulas (8) and (9), where the case in point is the instrumentation for measuring rotation frequencies and static influence coefficients.

\section{CONCLUSIONS}

We have studied the stability and accuracy of the algorithm developed for identification of eccentricities using static influence coefficients. The physical meaning of the condition number in the eccentricity identification problem is revealed. Optimal relationships are established between the balancing accuracy, the precision of the measurement instrumentation, and the dynamic model of the system under consideration.

\section{REFERENCES}

1. Royzman, V. and Bohorosh, A., Dynamic characteristics identification of aircraft enginebymethod of trial parameters, Mechanika, 2005, no. 3 (53), pp. 34-38.

2. Bakhvalov, N.S., Zhidkov, N.P., and Kobel'kov, G.M., Chislennye metody. Analiz, algebra, obyknovennye differentsial'nye uravneniya (Numerical Methods. Analysis, Algebra, Ordinary Differential Equations), Moscow: Nauka, 1973.

3. Levit, M.E., Roizman, V.P., et al., Osnovy balansirovochnoi tekhniki (Foundations of Balancing Equipment), vol. 2: Uravnoveshivanie gibkikh rotorov $i$ balansirovochnoe oborudovanie (Flexible Rotors Equilibring and Balancing Equipment), Shchepetil'nikov, V.A., Ed., Moscow: Mashinostroenie, 1975.

4. Goroshko, A.V. and Roizman, V.P., Shlyakhi pidvishchennya tochnosti rozv'yazkiv zvorotnikh zadach, Visn. Khmel'nits'k. Nats. Univ., 2013, no. 6, pp. 60-69.

5. Banakh, L.Ya. and Kempner, M.L., Vibrations of Mechanical Systems with Regular Structure, Springer, 2010.

Translated by S. Kittell 